\title{
Robust Fault-Tolerant Control for Descriptor Systems
}

\author{
B. Marx, D. Koenig, and D. Georges
}

\begin{abstract}
A new architecture for fault tolerant controllers is proposed for the generic class of descriptor systems. It is based on coprime factorization of nonproper systems and on the Youla parameterization of stabilizing controllers. Noticing that the Youla controllers include a so called residual signal, fault tolerant control is achieved. Nominal control and robust fault tolerance are addressed separately. Moreover, fault tolerant control can be improved with a scheme integrating fault diagnosis. The design of the diagnosis and fault tolerant control filters reduce to a standard $\boldsymbol{H}_{\infty}$-control problem of usual state-space system.
\end{abstract}

Index Terms-Coprime factorization, descriptor systems, robust fault tolerant control, Youla parameterization.

\section{INTRODUCTION}

Since systems are more and more complex, fault diagnosis and fault tolerant control have become challenging problems in the area of modern control theory; see [1] and [11]. Recently, efforts have been provided to integrate diagnosis in the controller design; see [12], [15].

In order to take into consideration physical constraints or static relations and more generally impulsive behaviors caused by an improper transfer matrix, the descriptor formulation (i.e., $E \dot{x}=A x+\cdots$ ) appears in many fields of system design and control; see [3] and [8]. Concerning the fault diagnosis problems, few results have been generalized to the descriptor case. In [11, Ch. 5], fault detection is based on observers, and unknown input observers are studied in [4]. In [7], fault detection and isolation is considered in the $H_{\infty}$-filtering framework and in [9], diagnosis is performed via coprime factorization of the nominal plant. However, none of these contributions envisaged fault tolerant control.

This note aims at generalizing fault tolerant control proposed by [12] to descriptor systems. Using the Youla parameterization, it is possible to address the fault diagnosis (FD), the control and the fault tolerant control (FTC) in distinct steps but in an unified approach. In the Youla parameterization of the stabilizing controllers [14], an inner signal appears to be a residual and can be filtered to perform robust fault diag-

Manuscript received January 30, 2004. Recommended by Associate Editor E. Bai

B. Marx was with the Laboratoire d'Automatique de Grenoble (UMR CNRS-INPG-UJF), 38402 Saint Martin d'Hères, Cedex, France. He is now with the Centre de Recherche en Automatique de Nancy, Institut National Polytechnique de Lorraine, 54516 Vandoeuvre-lŁs-Nancy Cedex, France (e-mail: benoit.marx@ensem.inpl-nancy.fr).

D. Koenig and D. Georges are with the Laboratoire d'Automatique de Grenoble (UMR CNRS-INPG-UJF), 38402 Saint Martin d'Hères, Cedex, France.

Digital Object Identifier 10.1109/TAC.2004.835595 
nosis. Moreover, the residual signal can be exploited for FTC by minimizing the output deviation caused by the fault and disturbance signals. To improve the performance of the FTC system, filters devoted to each fault (or combination of faults), should be synthesized and the appropriate filter is selected online according to the direction of the residual signal. It is important to note that, although descriptor systems may be improper, the design of the diagnosis and fault tolerant filters reduces to standard $H_{\infty}$-control for usual systems. Moreover, contrary to most residual generation or internal model methods, the matrix transfer of the process is not duplicated in the controller, thus due to the coprime factorization only proper filters are implemented, which is the major interest of this approach.

The note is organized as follows. Section II recalls some basics about descriptor systems and coprime factorization. Fault tolerant control is tackled in Section III. Before concluding, an example is provided.

\section{PRELIMINARIES}

In this section, some basics about descriptor systems are reminded, mainly taken from [3], a particular attention is paid to the coprime factorization which is the core of our approach.

Let us consider a linear time-invariant (LTI) descriptor system subject to fault and disturbance given by

$$
\left\{\begin{array}{l}
E \dot{x}(t)=A x(t)+B u(t)+R_{1} f(t)+E_{1} d(t) \\
y(t)=C x(t)+D u(t)+R_{2} f(t)+E_{2} d(t)
\end{array}\right.
$$

where $x \in \mathbb{R}^{n}$ is the descriptor variable, $u \in \mathbb{R}^{n} u$ is the control input, $y \in \mathbb{R}^{m}$ is the measured output, $d \in \mathbb{R}^{n} d$ is the disturbance, $f \in$ $\mathbb{R}^{n} f$ is the fault and $E, A, B, C, D, E_{1}, E_{2}, R_{1}$, and $R_{2}$ are known real constant matrices with compatible dimensions. As discussed in [5], the unknown vector $d(t)$ in the (1) embraces model uncertainties, additive perturbation, input, and output multiplicative perturbation and the vector $f(t)$ stands for dysfunctions, actuator, or sensor faults.

The matrix $E$ may be rank deficient: $\operatorname{rank}(E)=r \leq n$. The system (1) has an unique solution, for any initial condition, if it is regular (i.e., $\operatorname{det}(s E-A) \neq 0)$. Let note $q=\operatorname{deg} \operatorname{det}(s E-A)$. (1) has $q$ finite dynamic modes, $(n-r)$ static modes and $(r-q)$ impulsive modes. The finite modes correspond to the finite eigenvalues of the pencil matrix $(E, A)$. The system is called stable if and only if the finite modes are stable, i.e., the finite eigenvalues of $(E, A)$ lie in the open left halfplane. The impulsive modes may cause impulse terms in the response and thus are highly undesirable. A system has no impulsive mode and is said to be impulse free if and only if $\operatorname{deg}(\operatorname{det}(s E-A))=\operatorname{rank}(E)$. Since the transfer matrix of any impulse free descriptor system is (non strictly) proper it can be realized by an usual state-space representation $(A, B, C, D)$.

A descriptor system is impulse observable (respectively, $R$-detectable) if and only if it satisfies (2) [respectively, (3)]

$$
\begin{aligned}
\operatorname{rank}\left[\begin{array}{ccc}
E^{T} & 0 & 0 \\
A^{T} & E^{T} & C^{T}
\end{array}\right] & =n+\operatorname{rank} E \\
\operatorname{rank}\left[\begin{array}{c}
s E-A \\
C
\end{array}\right] & =n \\
\forall s & \in \mathbb{C} \quad \text { with } \Re(s) \geq 0 .
\end{aligned}
$$

If (2) is verified, there exists a matrix gain $L$ such that the pencil matrix $(E, A+L C)$ is impulse free. If (3) is verified, the unstable finite eigenvalues of $(E, A+L C)$ can be arbitrarily placed by the matrix gain $L$. If (3) is verified for all $s$, all the finite eigenvalues of $(E, A+L C)$ can be arbitrarily placed, and the system is called $R$-observable. Dual notions are defined for the controllability [3]. If $(E, A)$ is stable and impulse free, it is called admissible.
In the remainder of this note, the only necessary assumptions are the following.

A1) $(E, A, C)$ is impulse observable and detectable.

A2) $(E, A, B)$ is impulse controllable and stabilizable.

A3) $(E, A)$ is regular.

The LTI descriptor system (1) can also be described by $y(s)=G_{u}(s)$. $u(s)+G_{f}(s) \cdot f(s)+G_{d}(s) \cdot d(s)$ where $G_{u}(s)=C(s E-A)^{-1} B+$ $D, G_{d}(s)=C(s E-A)^{-1} E_{1}+E_{2}$, and $G_{f}(s)=C(s E-A)^{-1} R_{1}+$ $R_{2}$. A coprime factorization of the system (1) and of a stabilizing controller $K_{0}(s)$ is given by

$$
\begin{aligned}
G_{u} & =N_{u} M_{u}^{-1}=\tilde{M}_{u}^{-1} \tilde{N}_{u} \\
G_{f} & =N_{f} M_{f}^{-1}=\tilde{M}_{f}^{-1} \tilde{N}_{f} \\
G_{d} & =N_{d} M_{d}^{-1}=\tilde{M}_{d}^{-1} \tilde{N}_{d} \\
K_{0} & =U V^{-1}=\tilde{V}^{-1} \tilde{U}
\end{aligned}
$$

where the transfer matrices in (4) and (6) should satisfy the following double Bezout equation:

$$
\begin{aligned}
{\left[\begin{array}{ll}
I & 0 \\
0 & I
\end{array}\right] } & =\left[\begin{array}{cc}
\tilde{V} & -\tilde{U} \\
-\tilde{N}_{u} & \tilde{M}_{u}
\end{array}\right]\left[\begin{array}{cc}
M_{u} & U \\
N_{u} & V
\end{array}\right] \\
& =\left[\begin{array}{cc}
M_{u} & U \\
N_{u} & V
\end{array}\right]\left[\begin{array}{cc}
\tilde{V} & -\tilde{U} \\
-\tilde{N}_{u} & \tilde{M}_{u}
\end{array}\right]
\end{aligned}
$$

Let $K_{0}(s)$ be an observer-based feedback controller defined by

$$
\left\{\begin{array}{l}
E \dot{\hat{x}}_{c}=A \hat{x}_{c}+B u+L\left(C \hat{x}_{c}+D u-y\right) \\
u=F \hat{x}_{c}
\end{array}\right.
$$

or, equivalently

$$
K_{0}(s)=\left\{E,\left[\begin{array}{c|c}
A+L C+B F+L D F & -L \\
\hline F & 0
\end{array}\right]\right\}
$$

where the matrices $L$ and $F$ ensure the admissibility of $(E, A+L C)$ and $(E, A+B F)$, respectively. The matrices in (4) and (6) can be defined by [9]

$$
\begin{gathered}
{\left[\begin{array}{cc}
\tilde{V} & -\tilde{U} \\
-\tilde{N}_{u} & \tilde{M}_{u}
\end{array}\right]=\left\{E,\left[\begin{array}{c|cc}
A+L C & -(B+L D) & L \\
\hline F & I & 0 \\
C & -D & I
\end{array}\right]\right\}} \\
{\left[\begin{array}{cc}
M_{u} & U \\
N_{u} & V
\end{array}\right]=\left\{E,\left[\begin{array}{c|cc}
A+B F & B & -L \\
\hline F & I & 0 \\
C+D F & D & I
\end{array}\right]\right\} .}
\end{gathered}
$$

The transfer matrices $N_{f}, \tilde{N}_{f}, M_{f}, \tilde{M}_{f}, N_{d}, \tilde{N}_{d}, M_{d}$, and $\tilde{M}_{d}$ in (5), (6), (7) are easily deduced from (11) and (12). Moreover, a key point is that $\tilde{M}_{d}=\tilde{M}_{f}=\tilde{M}_{u}=\tilde{M}$ holds. Since the matrices $L$ and $F$ are chosen such that $(E, A+L C)$ and $(E, A+B F)$ are admissible, all the transfer matrices $M_{k}, N_{k}, \tilde{M}_{k}$, and $\tilde{N}_{k}$ are proper for $k \in\{u, d, f\}$. The impulsive terms in $G_{u}(s), G_{f}(s)$, and $G_{d}(s)$ are caused by their inverse $M_{k}^{-1}$ or $\tilde{M}_{k}^{-1}$, which may be strictly improper. System (1) can thus be written as

$$
y=\tilde{M}^{-1}\left(\tilde{N}_{u} u+\tilde{N}_{d} d+\tilde{N}_{f} f\right) .
$$

The set of all stabilizing controllers is given in [13].

Lemma 1: The set of all stabilizing controllers for $G_{u}(s)$ is given by $K(s)=\left(M_{u} Q+U\right)\left(N_{u} Q+V\right)^{-1}$ or, equivalently, $K(s)=$ $\left(\tilde{V}+Q \tilde{N}_{u}\right)^{-1}(\tilde{U}+Q \tilde{M})$, where $Q$ is an arbitrary proper stable transfer matrix. 


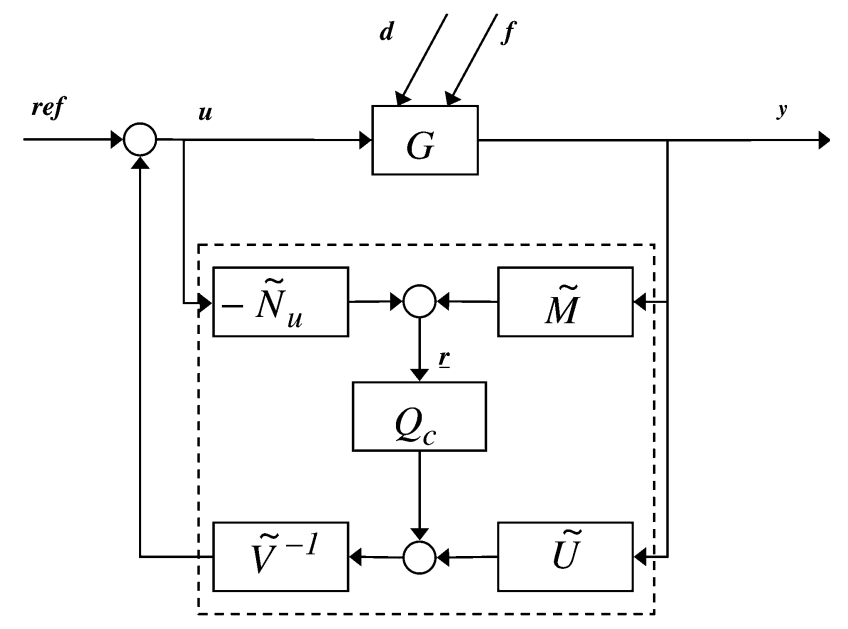

Fig. 1. Scheme of fault tolerant control.

\section{FAUlt TOlerant CONTROL}

In this section, an architecture of fault tolerant controllers is proposed for descriptor systems. On the one hand, nominal control performance, such as admissibility and pole placement of the nominal closed-loop system, are targeted. On the other hand, the deviation from the nominal response caused by the exogenous inputs $d(s)$ and $f(s)$ is minimized to achieve fault tolerant control. The proposed controller structure is depicted in Fig. 1. One should recognize the Youla parameterized controller.

This structure is interesting since, from (13), the internal signal, $\underline{r}$, appears to be a residual signal

$$
\begin{aligned}
\underline{r}(s) & =\tilde{M}(s)(s) y(s)-\tilde{N}_{u}(s) u(s) \\
& =\tilde{N}_{f}(s) f(s)+\tilde{N}_{d}(s) d(s) .
\end{aligned}
$$

Thus, fault tolerance (FT) aspects can easily be taken into consideration. Moreover, the reference signal "ref" does not impact on the residual generation. The response of the closed-loop system is given by (13), where $u$ is defined by

$$
\begin{aligned}
& u=\tilde{V}^{-1}\left(\tilde{U} y+Q_{c}\left(\tilde{M} y-\tilde{N}_{u} u\right)\right)+\text { ref } \\
& u=\tilde{V}^{-1}\left(\tilde{U} y+Q_{c}\left(\tilde{N}_{d} d+\tilde{N}_{f} f\right)\right)+\text { ref }
\end{aligned}
$$

combining (13) and (16), the response of the closed-loop system is given by $y=\left(\tilde{M}-\tilde{N}_{u} \tilde{V}^{1} \tilde{U}\right)^{-1}\left(\tilde{N}_{u}\right.$ ref $+\left(I+\tilde{N}_{u} \tilde{V}^{-1} Q_{c}\right)\left(\tilde{N}_{d} d+\right.$ $\left.\tilde{N}_{f} f\right)$ ). From (8) and matrix inversion formulas, one can derive that $V=\left(\tilde{M}-\tilde{N}_{u} \tilde{V}^{1} \tilde{U}\right)^{-1}$ and $V\left(I+\tilde{N}_{u} \tilde{V}^{-1} Q_{c}\right)=V+N_{u} Q_{c}$, and then finally obtain

$$
y=V \tilde{N}_{u} \operatorname{ref}+\left(V+N_{u} Q_{c}\right)\left(\tilde{N}_{d} d+\tilde{N}_{f} f\right) .
$$

From (17), it is clear that, on the one hand, the nominal control performances are set by $V \tilde{N}_{u}$, thus by $L$ and $F$ and, on the other hand, the fault tolerance is obtained by the appropriate choice of $Q_{c}(s)$. When no exogenous signal enter the system, the inner loop is inactive since $\underline{r}(s)=0$. Consequently, the choice of $Q_{c}(s)$ does not affect the nominal performance of the controller. Thus, nominal control and fault tolerance are addressed separately.

The controller is not necessary implemented as shown on Fig. 1 since it involves high order controller. Nevertheless, this formalism is appealing, not only for the sake of clarity, but also in the case of online reconfiguration of the controller, when $Q_{c}$ is monitored accordingly to a fault diagnosis filter (see Section III-C).

\section{A. Nominal Control}

It is readily verified that the nominal response corresponds to an observer based controller since

$$
\tilde{V}^{-1} \tilde{U}=\left\{E,\left[\begin{array}{c|c}
A+B F+L C+L D F & -L \\
\hline F & 0
\end{array}\right]\right\}
$$

and the closed-loop response is given by

$$
V \tilde{N}_{u}=\left\{\left[\begin{array}{cc}
E & 0 \\
0 & E
\end{array}\right],\left[\begin{array}{cc|c}
A+B F & -L C & -L D \\
0 & A+L C & B+L D \\
\hline C+D F & C & D
\end{array}\right]\right\}
$$

where the well-known separation principle holds. The admissibility of the closed-loop system is secured by A1) and A2), moreover, the finite dynamics of the closed-loop system can be arbitrarily chosen, provided $(E, A, B)$ and $(E, A, C)$ are $R$-controllable and $R$-observable respectively. The temporal characteristics of the response can be fixed by selecting $F$ and $L$ to ensure pole clustering of the closed-loop system. As introduced in [2], the concept of LMI region is an efficient tool to describe every convex region of the complex plane, which is symmetric with respect to the real axis, by two matrices $\alpha$ and $\beta$ (e.g., the left half-plane is defined by $\alpha=0$ and $\beta=1$ ). The LMI characterization of pole-clustering in LMI region for descriptor systems is treated in [10], and as a result $F$ and $L$ can be determined by solving strict LMI in order to ensure the pole clustering of the closed-loop system.

Theorem 1: For a given LMI region $D$, of the left half-plane defined by $\alpha$ and $\beta$, there exist $L$ and $F$ such that the closed-loop system (19) is $D$-admissible (i.e., is impulse free and has its finite pole in $D$ ), if and only if there exist symmetric positive definite matrices $P_{F}$ and $P_{L} \in \mathbb{R}^{n \times n}$, and matrices $S_{F}$ and $S_{L} \in \mathbb{R}^{(n-r) \times(n-r)}, H_{F} \in$ $\mathbb{R}^{n_{u} \times(n-r)}, H_{L} \in \mathbb{R}^{m \times(n-r)}, L_{F} \in \mathbb{R}^{n_{u} \times(n-r)}$, and $L_{L} \in \mathbb{R}^{m \times n}$ such that

$$
\begin{aligned}
& {\left[\alpha_{k l} E P_{F} E^{T}+\beta k l\left(A P_{F} E^{T}+B L_{F} E^{T}\right)+B H_{F} U^{T}\right.} \\
& \quad+A V S_{F} U^{T}+\beta l k\left(E P_{F} A^{T}+E L_{F}^{T} B^{T}\right) \\
& \left.\quad+U S_{F}^{T} V^{T} A^{T}+U H_{F}^{T} B^{T}\right]_{1 \leq k, l \leq p}<0 \\
& {\left[\alpha_{k l} E^{T} P_{L} E+\beta k l\left(A^{T} P_{L} E+C^{T} L_{L} E\right)+C^{T} H_{L}^{T} V^{T}\right.} \\
& \quad+A^{T} U S_{L} V^{T}+\beta l k\left(E^{T} P_{L} A+E^{T} L_{L}^{T} C\right) \\
& \left.+V S_{L}^{T} U^{T} A+V H_{L} C\right]_{1 \leq k, l \leq p}^{<0}
\end{aligned}
$$

where the notation $M=\left[M_{k l}\right]_{1 \leq k, l \leq m}$ means that $M$ is an $m \times$ $m$ block matrix with generic block $M_{k l} . U$ and $V$ are of full-column rank and are composed of bases of $\operatorname{Ker}(E)$ and $\operatorname{Ker}\left(E^{T}\right)$, respectively. Then, $F$ and $L$ are given by

$$
\begin{aligned}
& F=\left(L_{F} E^{T}+H_{F} U^{T}\right)\left(P_{F} E^{T}+V S_{F} U^{T}\right)^{-1} \\
& L=\left(P_{L} E+U S_{L} V^{T}\right)^{-T}\left(L_{L} E+H_{L} V^{T}\right)^{T} .
\end{aligned}
$$

Proof: This result is easily deduced from [10, Th. 1].

\section{B. Fault Tolerance}

In the absence of any fault and disturbance, the reconfiguration loop is inactive and does not affect the performance of the nominal closedloop system. In the presence of an exogeneous signal, $Q_{c}(s)$ provides a corrective term in order to compensate the effects of the exogenous signals. Indeed, it can be seen as an internal model based controller, with the major difference that $\tilde{M}(s)$ and $\tilde{N}_{u}(s)$ are always impulse 


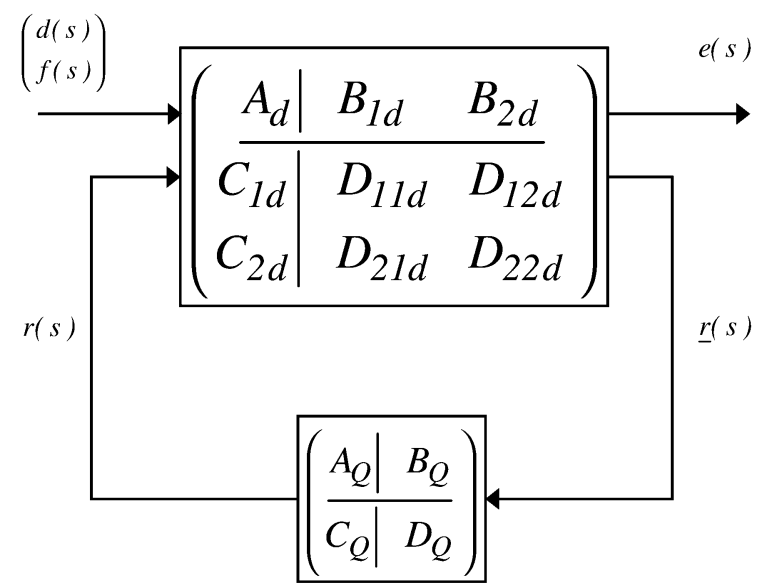

Fig. 2. Equivalent standard $H_{\infty}$ control problem.

free, even for impulsive nominal plants. From (17) a natural solution to FTC problem is to synthesize the parameter $Q_{c}(s)$ in order to minimize the $H_{\infty}$-norm of the transfer matrix from $d$ and $f$ to $y$, thus to minimize the criterion (24)

$$
J_{c}=\left\|\left(V+N_{u} Q_{c}\right)\left[\begin{array}{ll}
\tilde{N}_{d} & \tilde{N}_{f}
\end{array}\right]\right\|_{\infty} .
$$

All the factors in (24) are proper and minimizing the criterion $J_{c}$ reduces to the model matching problem of minimizing $\left\|T_{1}+T_{2} Q_{c} T_{3}\right\|_{\infty}$, where $T_{1}, T_{2}$, and $T_{3}$ are given by

$$
\begin{aligned}
& T_{1}=\left\{\left[\begin{array}{ll}
E & 0 \\
0 & E
\end{array}\right]\right. \\
& {\left.\left[\begin{array}{cc|cc}
A+B F & -L C & -L E_{2} & -L R_{2} \\
0 & A+L C & E_{1}+L E_{2} & R_{1}+L R_{2} \\
\hline C+D F & C & E_{2} & R_{2}
\end{array}\right]\right\} } \\
& T_{2}=\left\{E,\left[\begin{array}{l|l}
A+B F & B \\
\hline C+D F & D
\end{array}\right]\right\} \\
& T_{3}=\left\{E,\left[\begin{array}{l|ll}
A+L C & E_{1}+L E_{2} & R_{1}+L R_{2} \\
\hline C & E_{2} & R_{2}
\end{array}\right]\right\} .
\end{aligned}
$$

Since $T_{1}, T_{2}$, and $T_{3}$ are impulse free, these transfer matrices can be realized by usual state-space systems, let note $\left(A_{i}, B_{i}, C_{i}, D_{i}\right)$ a minimal realization of $T_{i}$, for $i \in\{1,2,3\}$. The minimization of $J_{c}$ can be formulated in the standard $H_{\infty}$ framework as finding the controller $Q_{c}$ that minimizes the $H_{\infty}$-norm of the closed-loop system depicted on Fig. 2, where the system $\left(A_{c}, B_{1 c}, B_{2 c}, C_{1 c}, C_{2 c}, D_{11 c}, D_{12 c}, D_{21 c}, D_{22 c}\right)$, is defined by

$$
\begin{aligned}
A_{c} & =\operatorname{diag}\left(A_{1}, A_{2}, A_{3}\right) \quad B_{1 c}=\left[\begin{array}{lll}
B_{1}^{T} & 0 & B_{3}^{T}
\end{array}\right]^{T} \\
B_{2 c} & =\left[\begin{array}{lll}
0 & B_{2}^{T} & 0
\end{array}\right]^{T} \\
C_{1 c} & =\left[\begin{array}{lll}
C_{1} & C_{2} & 0
\end{array}\right] \\
C_{2 c} & =\left[\begin{array}{lll}
0 & 0 & C_{3}
\end{array}\right] \quad D_{11 c}=D_{1} \quad D_{12 c}=D_{2} \\
D_{21 c} & =D_{3} \quad \text { and } D_{22 c}=0 .
\end{aligned}
$$

The LMI-based solution of [6] can be applied, provided $\left(A_{c}, B_{2 c}, C_{2 c}\right)$ is stabilizable and detectable, and provided the direct transfer from the control input to the measured output is null. These necessary conditions

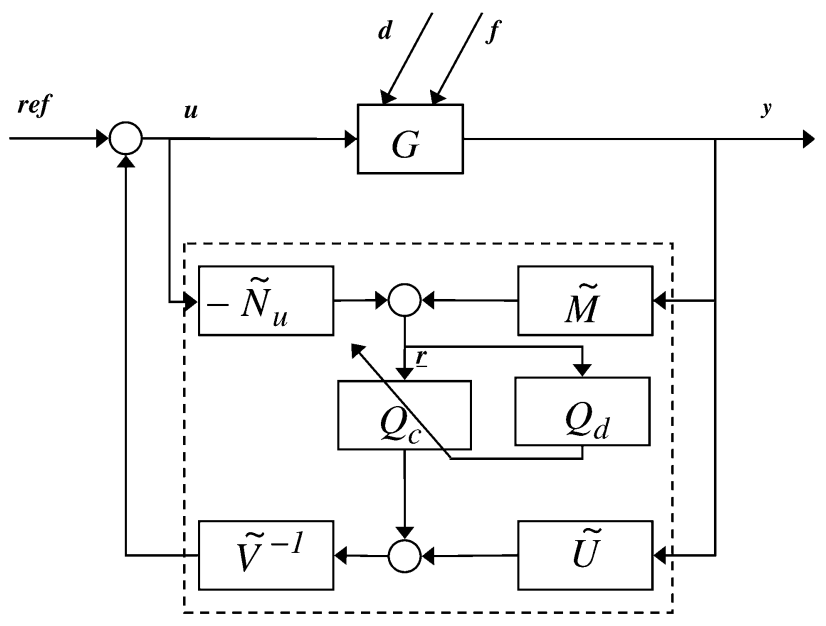

Fig. 3. Scheme of improved fault tolerant control.

are verified since $(E, A+B F)$ and $(E, A+L C)$ are admissible, thus stable, and since $D_{22 c}=0$, respectively.

Remark 1: A weighting function can be added in the criterion to enhance the robustness at high frequency or to put an emphasis on a particular frequency range if the power spectrum of the fault and disturbance is known.

Remark 2: The design of reduced order controller is highly encouraged since $A_{c}$ is a $(4 r \times 4 r)$ matrix. The following algorithm summarizes the fault tolerant control process.

Algorithm 1: To implement the fault tolerant controller

1) solve (20)-(21), to find $L$ and $F$ such that the nominal closedloop system is $D$-admissible;

2) find $Q_{c}$ by solving the equivalent standard $H_{\infty}$ control problem for (28);

3) implement the optimally robust fault tolerant controller

$$
u(s)=\left(\tilde{V}+Q_{c} \tilde{N}_{u}\right)^{-1}((\tilde{U}+Q \tilde{M}) y(s)+\tilde{V} \operatorname{ref}(s)) .
$$

\section{Improving the FTC Robustness}

In the previous scheme, the control filter $Q_{c}$ was designed to obtain optimal tolerance faced to all the possible faults. Assuming that several faults do not occur at the same time, dedicated controllers can be designed. $n_{f}$ control filters $Q_{c i}$ are synthesized by minimizing the criterion

$$
J_{c i}=\left\|\left(V+N_{u} Q_{c i}\right)\left[\begin{array}{ll}
\tilde{N}_{d} & \tilde{N}_{f i}
\end{array}\right]\right\|_{\infty}
$$

where $\tilde{N}_{f i}$ is the $i$ th column of $\tilde{N}_{f}$. Another filter, dedicated to the fault-free case, is determined by minimizing the following criterion:

$$
J_{c 0}=\left\|\left(V+N_{u} Q_{c i}\right) \tilde{N}_{d}\right\|_{\infty} .
$$

Remark 3: If simultaneous faults may appear, a combination of fault should be considered, but the methodology remains.

This structure, depicted on Fig. 3, permits to significantly reduce the conservatism introduced by the $H_{\infty}$ design of $Q_{c}$. The selection of the appropriate control filter is done by a simple logic, exploiting the residual given by a diagnosis filter, $Q_{d}$, synthesized by standard $H_{\infty}$ techniques, presented in [9]. $Q_{d}$ is determined in order to shape the response of the filter to the faults, while minimizing its sensitivity to to the disturbance. This is achieved by minimizing the criterion

$$
J_{d}=\left\|\left[Q_{d} \tilde{N}_{d} \quad Q_{d} \tilde{N}_{f}-T\right]\right\|_{\infty}
$$



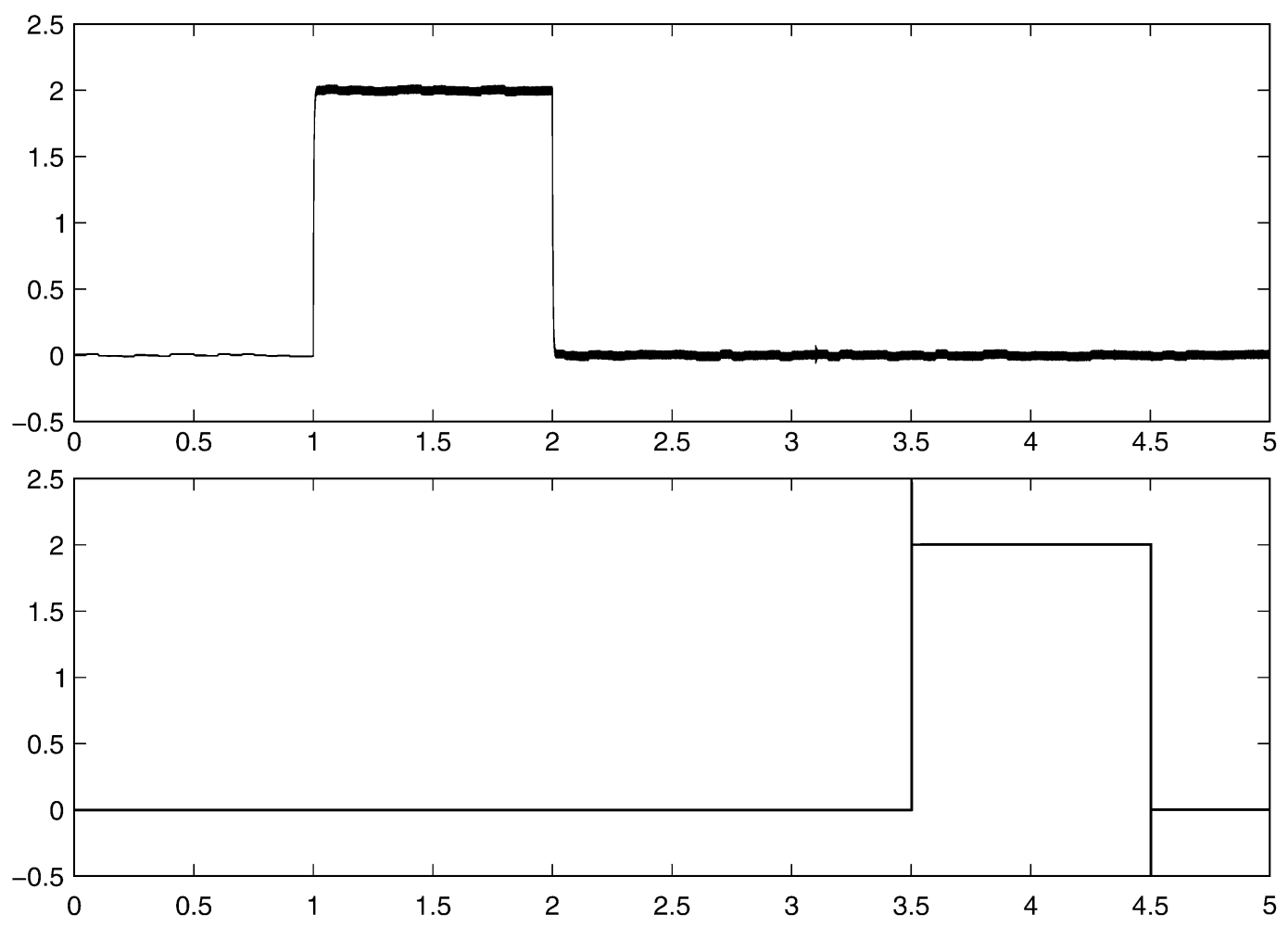

Fig. 4. Fault estimation.

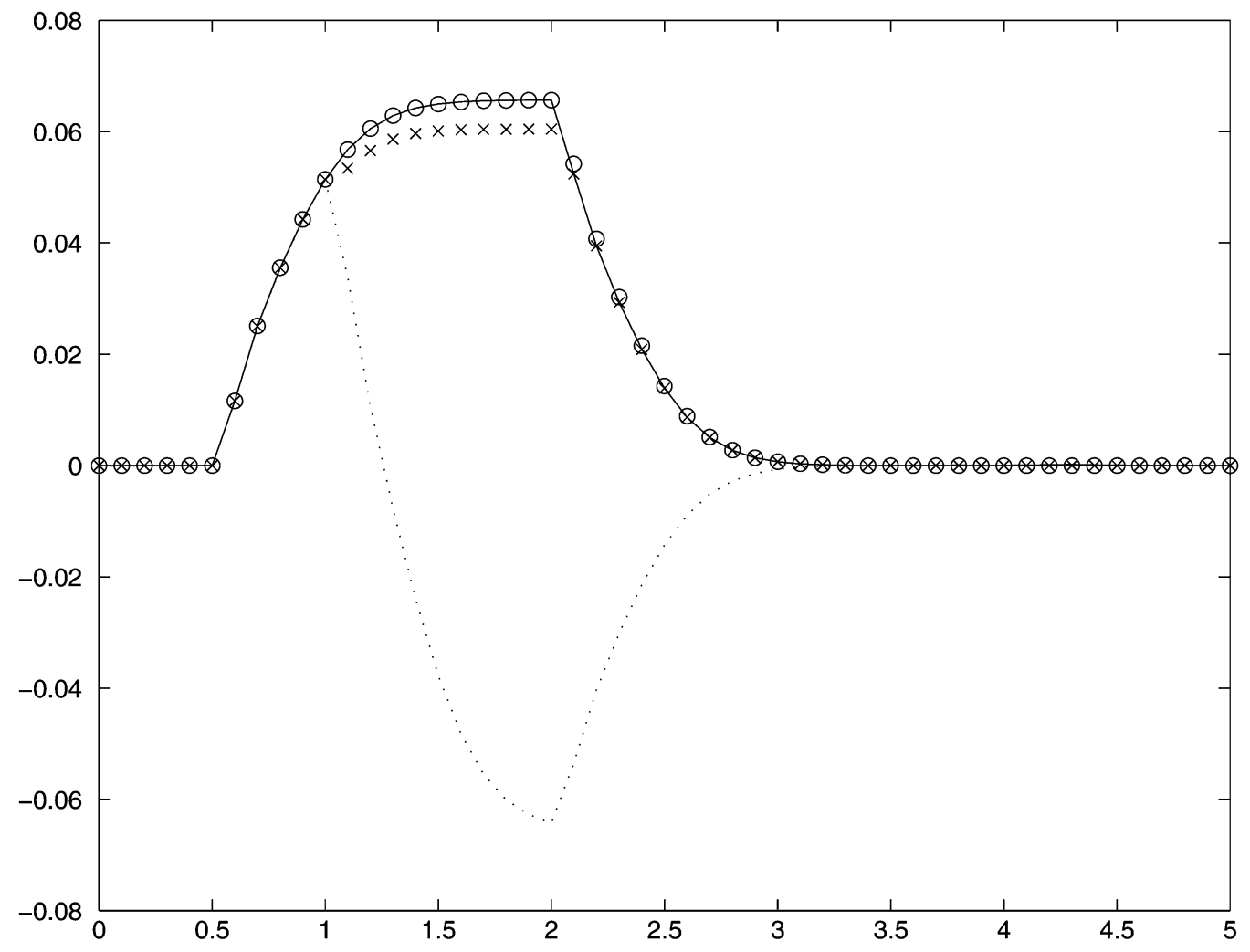

Fig. 5. Comparison of the obtained output $y_{1}(t)$ for different control methodologies.

where $T(s)$ is the desired frequency response to the faults. This model matching problem reduces to standard $H_{\infty}$ control problem for usual systems and can be addressed by LMI-based solution of [6], as pro- posed in [9]. Each component of the signal $r$ is compared with a fixed threshold. A natural threshold is the optimal $J_{d}$ obtained when synthesizing the diagnosis filter $Q_{d}$. 


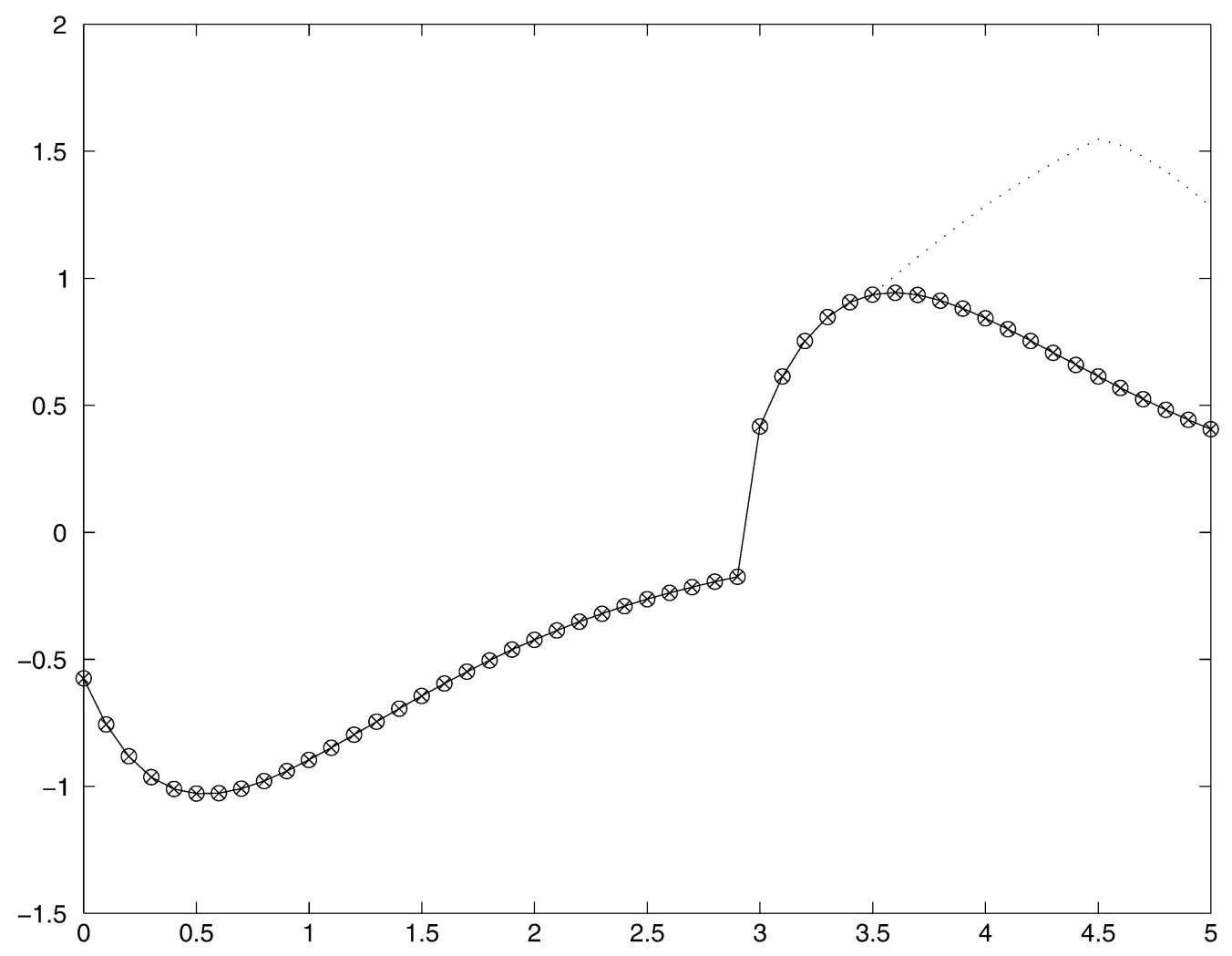

Fig. 6. Comparison of the obtained output $y_{2}(t)$ for different control methodologies.

\section{NUMERICAL EXAMPLE}

Let us consider (1), affected by an actuator biais $f_{1}(t)$, a sensor biais $f_{2}(t)$ and an unknown input $d(t)$. The exogenous signals are defined by $f_{1}(t)=\{2$, for $1 \leq t \leq 2,0$ else $\}, f_{2}(t)=\{2$, for $3.5 \leq$ $t \leq 4.5,0$ else $\}$ and $d(t)$ is a random number uniformly distributed in $[-1,1]$

$$
\begin{aligned}
& E=\left[\begin{array}{llll}
1 & 0 & 0 & 0 \\
0 & 1 & 0 & 0 \\
0 & 0 & 0 & 1 \\
0 & 0 & 0 & 0
\end{array}\right] \quad A=\left[\begin{array}{cccc}
-15 & 1 & 0 & 0 \\
5 & -10 & 0 & 0 \\
0 & 0 & 1 & 0 \\
0 & 0 & 0 & 1
\end{array}\right] \\
& B=\left[\begin{array}{ll}
1 & 0 \\
0 & 0 \\
0 & 0 \\
0 & 1
\end{array}\right] \quad E_{1}=\left[\begin{array}{llll}
0,01 & 0 & 0 & 0
\end{array}\right]^{T} \\
& R_{1}=\left[\begin{array}{cccc}
-1 & 0 & 0 & 0 \\
0 & 0 & 0 & 0
\end{array}\right]^{T} \quad C=\left[\begin{array}{llll}
0 & 1 & 0 & 0 \\
0 & 0 & 1 & 0
\end{array}\right] \\
& D=\left[\begin{array}{ll}
0 & 0 \\
0 & 0
\end{array}\right] \quad E_{2}=\left[\begin{array}{l}
0 \\
0
\end{array}\right]
\end{aligned}
$$

and

$$
R_{2}=\left[\begin{array}{ll}
0 & 0 \\
0 & 1
\end{array}\right] \text {. }
$$

One can check that the necessary assumptions are verified. Following the proposed methodology, we chose the nominal controller such that the real part of the closed-loop poles $\lambda_{i}$ verify $-10<\Re\left(\lambda_{i}\right)<-1$. The obtained results are displayed in Figs. 4-6. The estimation of the faults is shown on Fig. 4. Figs. 5 and 6 give the outputs $y_{1}(t)$ and $y_{2}(t)$ respectively in different cases. The disturbance and fault free, nominal response is represented with circles. The observer-based control affected by disturbance and fault is represented by the dashed lines. The FT control is represented by the crossed lines and the improved
FT control is represented by the solid lines. It is clearly seen, on both Figs. 5 and 6 that the observer-based controller does not match the fault and disturbance free case, whereas the proposed FT does. The improvment of the FTC obtained with adaptative controller appears in Fig. 5.

\section{CONCLUSION}

In this note, fault tolerant feedback control is extended to descriptor systems. The coprime factorization of descriptor systems permits to build a pre residual signal. Then different filters are synthesized, by standard $H_{\infty}$-techniques, to perform fault tolerant control. The fault tolerant controller is based on the well known Youla controller parameterization. The parameterizing filter is designed to minimize the deviation of the output caused by the fault and the disturbance. A high-performance FTC architecture includes a fault diagnosis filter to adapt online the controller parameter and thus improve the fault tolerance by selecting a controller dedicated to the appearing fault, and limit the conservatism introduced in the $H_{\infty}$ design of the FTC filter.

\section{REFERENCES}

[1] J. Chen and R. J. Patton, Robust Model-Based Fault Diagnosis for Dynamic Systems. Norwell, MA: Kluwer, 1999.

[2] M. Chilali and P. Gahinet, " $H_{\infty}$ design with pole placement constraints: An LMI approach," IEEE Trans. Automat. Contr., vol. 41, pp. 358-367, Mar. 1996.

[3] L. Dai, Singular Control Systems. Berlin, Germany: Springer-Verlag, 1989.

[4] G. R. Duan, D. Howe, and R. J. Patton, "Robust fault detection in descriptor linear systems via generalized unknown input observers," presented at the Triennal World Congr., Beijing, P.R. China, 1999.

[5] P. M. Frank and X. Ding, "Frequency domain approach to optimally robust residual generation and evaluation for model-based fault diagnosis," Automatica, vol. 30, pp. 789-804, 1994.

[6] P. Gahinet and P. Apkarian, "A linear matrix inequality approach to $H_{\infty}$ control," Int. J. Robust d Nonlinear Control, vol. 4, pp. 421-448, 1994. 
[7] D. Koenig, S. Mammar, and B. Marx, " $H_{\infty}$ fault detection and isolation for descriptor: A matrix inequalities approach," in Proc. Amer. Control Conf., vol. 2, 2002, pp. 1080-1081.

[8] F. L. Lewis, "A survey of linear singular systems," Circuits Syst. Signal Process., vol. 5, pp. 3-36, 1986.

[9] B. Marx, D. Koenig, and D. Georges, "Robust fault diagnosis for descriptor systems-A coprime factorization approach," in Proc. IFAC SAFEPROCESS'03, 2003, pp. 507-512.

[10] — - "Robust pole-clustering in LMI regions, a characterization via strict LMIs," in Proc. Eur. Control Conf., 2003, pp. 507-512.

[11] R. J. Patton, P. M. Frank, and R. N. Clark, Issues of Fault Diagnosis for Dynamical Systems. London, U.K.: Springer-Verlag, 2000

[12] J. Stoustrup and H. Niemann, "Fault tolerant feedback control," in Proc. Eur. Control Conf., 2001, pp. 1970-1974.

[13] K. Takaba, N. Morihira, and T. Katayama, " $H_{\infty}$-control for descriptor systems-A $J$-spectral factorization approach," in Proc. Conf. Decision Control, 1994, pp. 2251-2256.

[14] K. Zhou, J. C. Doyle, and K. Glover, Robust and Optimal Control. Upper Saddle River, NJ: Prentice-Hall, 1996.

[15] K. Zhou and Z. Ren, "A new controller architecture for high performance, robust, and fault-tolerant control," IEEE Trans. Automatic Control, vol. 46, pp. 1613-1618, Oct. 2001. 